\title{
Communications
}

\section{On Reconciling Ground-Based With Spaceborne Normalized Radar Cross Section Measurements}

François Baumgartner, Jens Munk, Kenneth C. Jezek, and Sivaprasad Gogineni

\begin{abstract}
This study examines differences in the normalized radar cross section, derived from ground-based versus spaceborne radar data. A simple homogeneous half-space model, indicates that agreement between the two improves as 1) the distance from the scatterer is increased; and/or 2) the extinction coefficient increases.
\end{abstract}

Index Terms-Ice, radar scattering, snow.

\section{INTRODUCTION}

The normalized radar cross section $\left(\sigma^{0}\right)$ is used to effectively describe backscatter from snow, under the assumption that the range to the scattering particles is accurately known. For spaceborne radar, where the total range is large compared to the firn penetration, this assumption is valid. However, for short-range radar, firn penetration depths are typically on the order of the antenna range. As a result, the normalized radar cross section derived from ground-based data is a function of antenna range, as well as firn physical properties.

Previously, ground-based and spaceborne radar backscatter have been reconciled by defining an effective range $R_{\text {eff }} \equiv R_{s}+\delta R$, where $R_{s}$ is the antenna range to the snow surface, as illustrated in Fig. 1 [1], [2]. The additional term $\delta R$ is an added range accounting for radar penetration into the scattering medium, which can be significant. The relative size of $\delta R$ is directly related to firn physical properties and is determined experimentally such that $\sigma^{0}$ does not vary with antenna height. In this communication, we describe an analytic approach for reconciling spaceborne and ground-based data that can also be used to estimate the extinction coefficient when both types of data are available.

Assuming a homogeneous half-space, we derive an expression for $\sigma^{0}$ as a function of antenna range and physical properties of the firn. Our relationship describes why differences in derived values for $\sigma^{0}$ occur between spaceborne and ground-based radar. Two sites (GITS and NASA-U) on the Greenland Ice Sheet are used to illustrate the observed differences in $\sigma^{0}$ for ground-based versus spaceborne radar.

\section{FORMULATION}

The power return resulting from an incremental volume is given by

$$
d^{3} P_{r}=\frac{P_{t} \lambda^{2}}{(4 \pi)^{3}} G_{0}^{2} g^{2}(\theta, \phi) \sigma_{v} \frac{e^{-2 \tau}}{R^{4}} d V
$$

Manuscript received October 10, 2000; revised July 16, 2001.

F. Baumgartner is with the Arctic Technology Center, Technical University of Denmark (DTU), 2800 Lyngby, Denmark (e-mail: frb@byg.dtu.dk).

J. Munk and K. C. Jezek are with the Byrd Polar Research Center, The Ohio State University, Columbus, OH 43210-1002 USA (e-mail: munk@ frosty.mps.ohio-state.edu; jezek@iceberg.mps.ohio-state.edu).

S. P. Gogineni is with the Radar Systems and Remote Sensing Laboratory, University of Kansas, Lawrence, KS 66045 USA (e-mail: gogineni@ittc.ukans.edu)

Publisher Item Identifier S 0196-2892(02)02127-7.

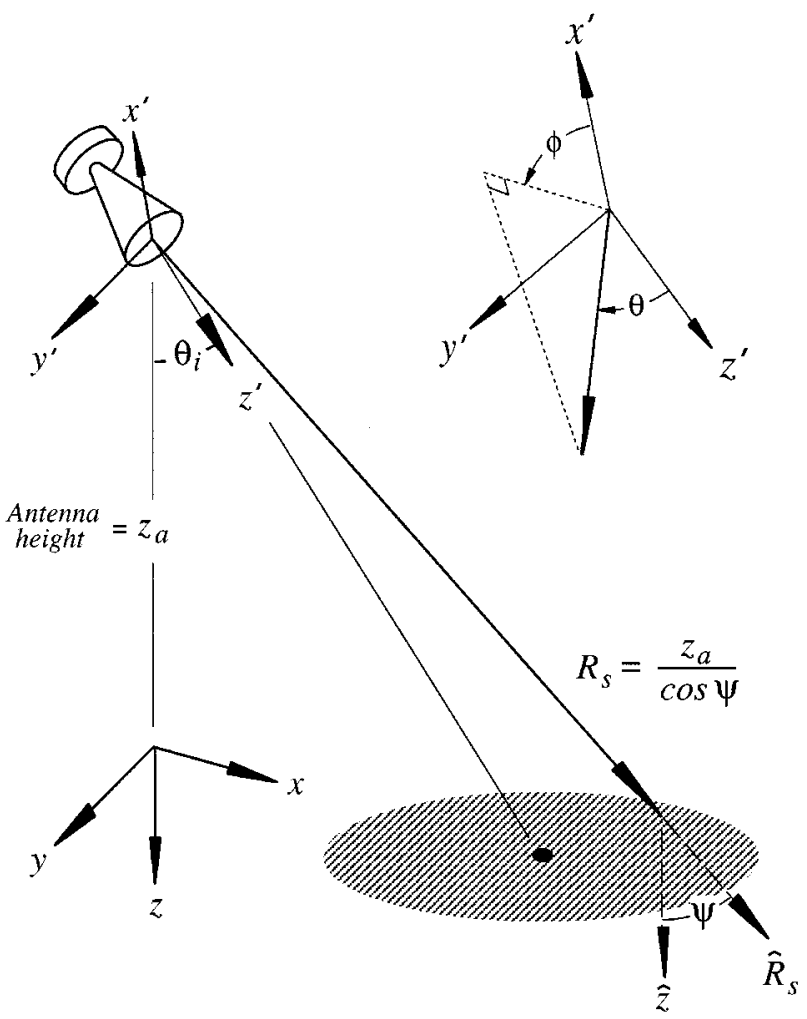

Fig. 1. Antenna at height $z_{a}$ illuminating the surface $z=0$, with the shaded region corresponding to the main antenna beam.

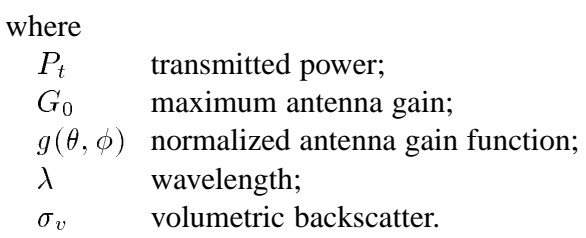

$R$ is the range from the antenna to the volume $d V=R^{2} d R d \Omega$, where $d \Omega \equiv \sin \theta d \theta d \phi$, and $\theta$ and $\phi$ are with respect to the primed coordinates, as shown in Fig. 1. The term $e^{-2 \tau}$ accounts for two-way attenuation within the scatterer where

$$
\tau \equiv \int_{R_{s}}^{R} \kappa_{e} d R
$$

where $\kappa_{e}$ is the extinction coefficient and $R_{s}$ is as previously defined. Losses within the scatterer are described by the extinction coefficient

$$
\kappa_{e}=\kappa_{a}+\kappa_{s}
$$

where $\kappa_{a}$ and $\kappa_{s}$ are the absorption and scattering coefficient, respectively [3]. In terms of the solid angle $d \Omega,(1)$ is expressed as

$$
d^{2} P_{r}=\frac{P_{t} \lambda^{2}}{(4 \pi)^{3}} G_{0}^{2} g^{2}(\theta, \phi) \gamma\left(R_{s}\right) d \Omega
$$

where

$$
\gamma\left(R_{s}\right) \equiv \int_{R_{s}}^{\infty} \sigma_{v} \frac{e^{-2 \tau}}{R^{2}} d R
$$


For a homogeneous half-space, $\sigma_{v}$ and $\kappa_{e}$ are constant, and (5) can be written as

$$
\gamma\left(R_{s}\right)=\sigma_{v} f\left(R_{s}\right)
$$

where

$$
f\left(R_{s}\right) \equiv \int_{R_{s}}^{\infty} \frac{e^{-2 \kappa_{e} \Delta R}}{R^{2}} d R
$$

and $\Delta R \equiv R-R_{s}$. Likewise, the power return can be expressed in terms of a projected surface $d A_{s}$, such that

$$
d^{2} P_{r}=\frac{P_{t} \lambda^{2}}{(4 \pi)^{3}} \frac{G_{0}^{2} g^{2}(\theta, \phi)}{R_{s}^{4}} \sigma^{0} d A_{s}
$$

where $\sigma^{0}$ is the normalized radar cross section. The incremental surface

$$
d A_{s} \equiv \frac{R_{s}^{2}}{\cos \psi} d \Omega
$$

where $\cos \psi=\hat{R}_{s} \cdot \hat{z}$ represents the localized incidence angle, with $\hat{R}_{s}=\mathbf{R}_{s} / R_{s}$, and $\hat{z}$ normal to the snow surface, as shown in Fig. 1. Substituting (9) into (8) yields

$$
d^{2} P_{r}=\frac{P_{t} \lambda^{2}}{(4 \pi)^{3}} \frac{G_{0}^{2} g^{2}(\theta, \phi)}{R_{s}^{2} \cos \psi} \sigma^{0} d \Omega
$$

which expresses $\sigma^{0}$ in terms of the solid angle $d \Omega$. Equating like terms in (4) and (10) yields

$$
\sigma^{0}=\sigma_{v} f\left(R_{s}\right) R_{s}^{2} \cos \psi
$$

which relates the normalized radar cross section as defined using an equivalent surface and volume backscatter coefficient. For spaceborne radar, $R_{s} \gg 1 / \kappa_{e}$ and (7) can be approximated as

$$
f\left(R_{s}\right) \approx \frac{1}{2 \kappa_{e}} \frac{1}{R_{s}^{2}}
$$

Then, assuming a pencil beam approximation [4]

$$
\sigma_{\infty}^{0}=\frac{\sigma_{v}}{2 \kappa_{e}} \cos \theta_{i}
$$

where $\sigma_{\infty}^{0}$ is the normalized radar cross section when $R_{s} \rightarrow \infty$, and $\theta_{i}$ is the antenna incidence angle, measured with respect to the $z$-axis (see Fig. 1).

As expressed in (11), $\sigma^{0}$ is a function of both the extinction coefficient $\kappa_{e}$, the antenna range $R_{s}$, and the local incidence angle $\psi$.

Since the volume scattering coefficient $\sigma_{v}$ is related only to the firn physical properties of the illuminated snow, (11) and (13) can be combined to yield

$$
\sigma^{0}=\left\{2 \kappa_{e} f\left(R_{s}\right) R_{s}^{2} \frac{\cos \psi}{\cos \theta_{i}}\right\} \sigma_{\infty}^{0}
$$

which relates the ground-based and spaceborne normalized radar cross section coefficients. For the same angle of incidence, and assuming a pencil beam approximation for the ground-based data, (14) yields

$$
\frac{\sigma^{0}}{\sigma_{\infty}^{0}}=2 \kappa_{e} f\left(R_{s}\right) R_{s}^{2}
$$

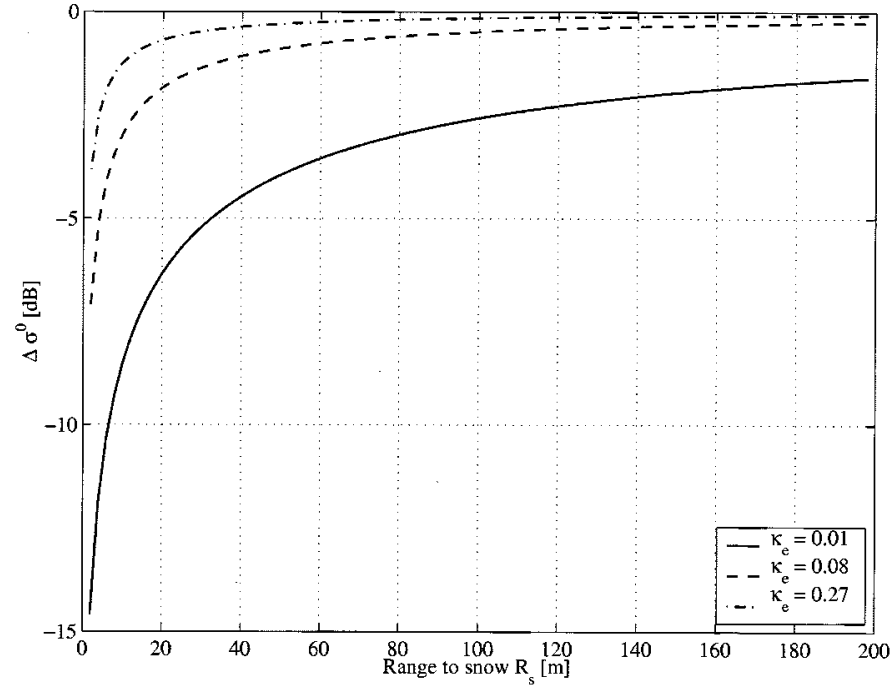

Fig. 2. Plot showing differences between ground-based and spaceborne derived normalized radar cross section $\Delta \sigma^{0}$ versus the antenna range $R_{s}$.

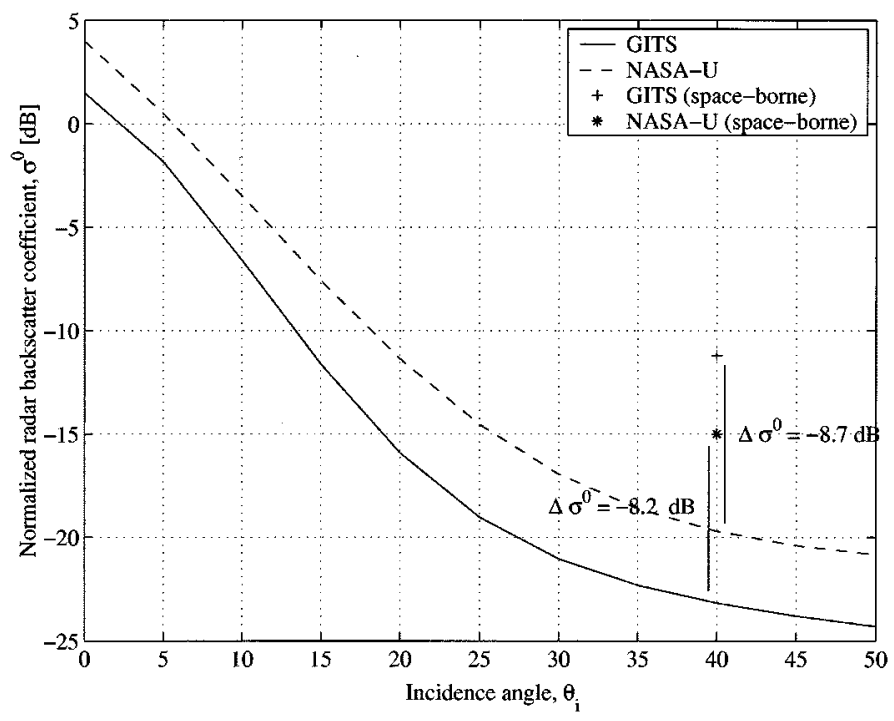

Fig. 3. Comparison of $\sigma^{0}$ derived from ground-based versus spaceborne data at GITS and NASA-U.

By definition, this ratio is less than unity, and hence the ground-based normalized radar cross section is always less that its spaceborne counterpart. Finally, from (12) and (15)

$$
\lim _{R_{s} \rightarrow \infty} \sigma^{0}=\sigma_{\infty}^{0}
$$

as it must.

\section{RESULTS}

Data from two sites on the Greenland Ice Sheet, GITS $\left(77^{\circ} 06^{\prime} \mathrm{N}\right.$, $\left.61^{\circ} 00^{\prime} \mathrm{W}\right)$ and NASA-U $\left(73^{\circ} 50^{\prime} \mathrm{N}, 49^{\circ} 30^{\prime} \mathrm{W}\right)$ were collected during May 21-25, 1995 [5]. The GITS and NASA-U sites are within the dry-snow zone and a transition zone between the dry-snow and percolation zone, respectively. No melt features were observed within the upper 7-8 $\mathrm{m}$ of firn at the NASA-U site. For the ground-based data, $\theta_{i}$ varied from 0 to $50^{\circ}$, in $5^{\circ}$ increments. The antenna height $z_{a}$, specified by the distance from the antenna feed to the firn surface (see Fig. 1), was 
TABLE I

PARAMETERS USED IN COMPARISON BETWEEN GROUND-BASEd VERSUS SPACEBORNe DERIVED NoRMALIZED RADAR CROSS SECTION

\begin{tabular}{|c|c|c|}
\hline & GITS & NA.SA-U \\
\hline & Ground-base & system \\
\hline$z_{a}$ & $1.67 \mathrm{~m}$ & $1.84 \mathrm{~m}$ \\
\hline$\theta_{i}$ & $0-50^{\circ}$ & $0-50^{\circ}$ \\
\hline${ }^{\dagger} R_{s}$ & $2.18 \mathrm{~m}$ & $2.40 \mathrm{~m}$ \\
\hline$\dagger_{\sigma^{0}}$ & $-23.6 \mathrm{~dB}$ & $-19.9 \mathrm{~dB}$ \\
\hline
\end{tabular}

ERS-1 Scatterometer

\begin{tabular}{ccc}
\multicolumn{3}{c}{ ERS-1 Scatterometer } \\
\hline \hline$\theta_{i}$ & $40^{\circ}$ & $40^{\circ}$ \\
$R_{s}$ & $\sim 1000 \mathrm{~km}$ & $\sim 1000 \mathrm{~km}$ \\
$\sigma_{\infty}^{0}$ & $-15.4 \mathrm{~dB}$ & $-11.2 \mathrm{~dB}$ \\
\hline
\end{tabular}

\begin{tabular}{ccc}
\multicolumn{3}{c}{ Derived quantities } \\
\hline \hline$\Delta \sigma^{0}$ & $-8.2 \mathrm{~dB}$ & $-8.7 \mathrm{~dB}$ \\
$\kappa_{e}$ & $0.0505 \mathrm{~m}^{-1}$ & $0.0398 \mathrm{~m}^{-1}$ \\
$d$ & $9.9 \mathrm{~m}$ & $12.5 \mathrm{~m}$ \\
\hline
\end{tabular}

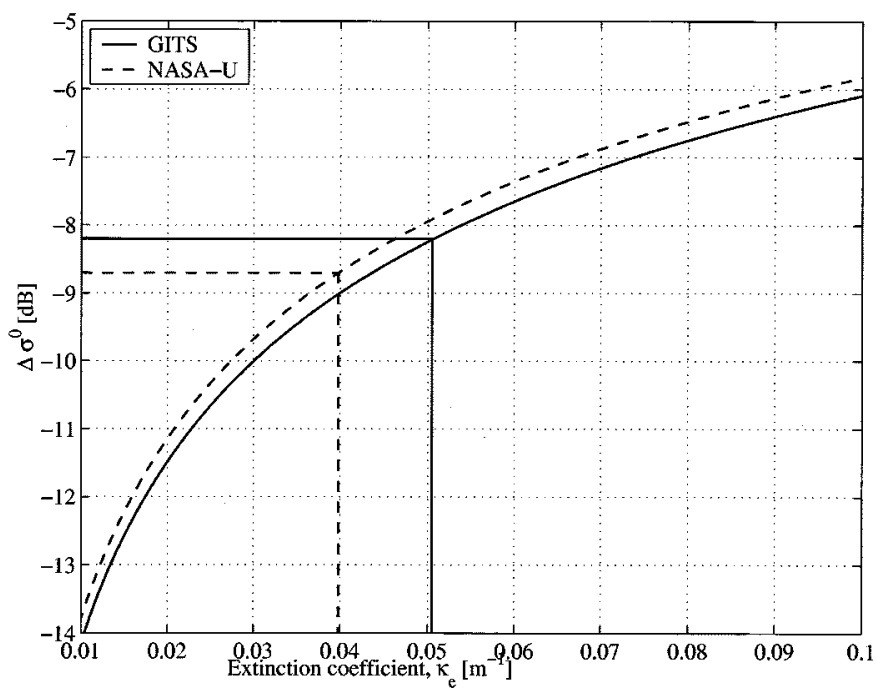

Fig. 4. Plot showing differences between ground-based and spaceborne derived normalized radar cross section $\Delta \sigma^{0}$ versus $\kappa_{e}$ for $\theta_{i}=40^{\circ}$, with $R_{s}$ corresponding to GITS and NASA-U.

1.67 and $1.84 \mathrm{~m}$ at GITS and NASA-U, respectively. Fig. 3 is a comparison between our in-situ data, adjusted to account for the antenna pattern, and ERS-1 scatterometer data, acquired during May 21-26, 1995 (JD 141-146), at C-band and for VV polarization. The scatterometer data were processed using a resolution enhancement technique [6], and have been "incidence angle-normalized" to $40^{\circ}$, from data covering a wide range of incidence angles. The normalized radar cross section ratio, given in (15), is plotted versus $R_{s}$ in Fig. 2, for $\kappa_{e}=$ $0.01,0.08$, and 0.27 and $\mathrm{m}^{-1}$, where, for notational convenience, we define $\Delta \sigma^{0} \equiv 10 \log _{10}\left(\sigma^{0} / \sigma_{\infty}^{0}\right)$. As shown in Fig. $2, \Delta \sigma^{0}$ increases as the antenna nears the illuminated surface, and as the extinction coefficient is decreased. Differences between the ground-based and spaceborne derived values of $\sigma^{0}$ result from the radar penetration within the firn relative to the antenna-snow surface range, which is significant for ground-based radar. Although our analysis assumes a homogeneous half-space, we speculate that ground-based radar will be more sensitive to near surface properties, while a spaceborne radar will effectively include backscatter from a more extensive range into the firn.

Table I summarizes information relevant in comparing the groundbased and spaceborne radar at the two sites. Curves shown in Fig. 4 were generated from (15), over a range of extinction coefficients, and with $R_{s}$ as given in Table I. The extinction coefficient is estimated using Fig. 4 and $\Delta \sigma^{0}$ (see Fig. 3). At GITS and NASA-U, we obtain $\kappa_{e}=0.0505$ and $0.0398 \mathrm{~m}^{-1}$, respectively. In terms of the two-way penetration depth $\left(d \equiv 1 / 2 \kappa_{e}\right)$, we obtain $d=9.9$ and $12.5 \mathrm{~m}$. These penetration depths are within the range obtained in other studies within the Greenland dry-snow zone $(d=7.5-9 \mathrm{~m})$ [7].

\section{CONCLUSIONS}

We have provided a plausible explanation for the observed differences between normalized radar cross section derived from groundbased versus spaceborne measurements, based on a unique definition for the normalized radar cross section.

Based on (15), which assumes a homogeneous half-space model, differences between ground-based and spaceborne derived normalized radar cross section increase as: 1) the range for the ground-based radar decreases, and 2) the extinction coefficient, which effects the penetration depth, is decreased.

These results are confirmed by analyzing the differences between ground-based and spaceborne measurements of $\sigma_{0}$ at GITS and NASA-U, in conjunction with (15).

\section{REFERENCES}

[1] H. Rott, K. Sturm, and H. Miller, "Active and passive microwave signatures of antarctic firn by means of field measurements and satellite data," Ann. Glaciol., vol. 17, pp. 337-343, 1993.

[2] H. Rott and K. Sturm, "Microwave signature measurements of antarctic and alpine snow," in Proc. 11th EARSeL Symp., Graz, Austria, 1991, pp. $140-151$.

[3] F. T. Ulaby, R. K. Moore, and A. K. Fung, Microwave Remote Sensing. Norwood, MA: Artech House, 1986, vol. III.

[4] - Microwave Remote Sensing. Reading, MA: Addison-Wesley, 1982, vol. II.

[5] F. Baumgartner, K. Jezek, R. R. Forster, S. P. Gogineni, and I. H. H. Zabel, "Spectral and angular ground-based backscatter measurements of Greenland snow facies," in Proc. IGARSS, June 1999, pp. 1053-1055.

[6] D. G. Long, P. J. Hardin, and P. T. Whiting, "Resolution enhancement of spaceborne scatterometer data," IEEE Trans. Geosci. Remote Sensing, vol. GE-25, no. 6, pp. 700-715, 1987.

[7] E. W. Hoen and H. A. Zebker, "Penetration depths inferred from interferometric volume decorrelation observed over the Greenland Ice Sheet," IEEE Trans. Geosci Remote Sensing, vol. 38, pp. 2571-2583, Nov. 2000. 